\title{
On hybrid intelligence-based control approach with its application to flexible robot system
}

\author{
E. Khoobjou ${ }^{1}$ and A. H. Mazinan ${ }^{2^{*}}$
}

\author{
*Correspondence: \\ mazinan@azad.ac.ir; \\ ahmazinan@gmail.com; \\ ah_mazinan@yahoo.com \\ ${ }^{2}$ Department of Control \\ Engineering, Faculty \\ of Electrical Engineering \\ South Tehran Branch, Islamic \\ Azad University (IAU), No. \\ 209, North Iranshahr Street, \\ P.O. Box 11365/4435, Tehran, \\ Iran \\ Full list of author information \\ is available at the end of the \\ article
}

\begin{abstract}
Flexible robot system is in general taken into real consideration as most important process in a number of academic and industrial environments. Due to the fact that the aforementioned system is so applicable in real domains, the novel ideas with respect to state-of-the-art in outperforming its performance are always valuable. With this purpose, a number of the soft computing techniques can be preferred with reference to the traditional ones to predict and optimize the overall performance of the abovecaptioned process. The approach proposed here is in fact organized in line with the integration of the fuzzy-based approach in association with the neural networks, in order to enable the process under control to be capable of learning and adapting to be matched, in a number of real environments. It can be shown that the outcomes tolerate the imprecise circumstances, as one of advantages regarding the fuzzy-based approach. In the present investigation, a new hybrid approach is proposed to deal with the arm of flexible robot system through the neural networks, the fuzzy-based approach and also the particle swarm optimization. It should be noted that the objective of the proposed research is to control the claw of robot system including twodegree-of-freedom movable arms. The results indicate that the mean-square error and the root-mean-square error are accurately outperformed with reference to the traditional ones, tangibly.
\end{abstract}

Keywords: Flexible robot system, Intelligence-based control approach, Particle swarm optimization

\section{Background}

The main idea of the soft computing is first introduced by Professor Zadeh, ${ }^{1}$ entitled What is Soft Computing?, published in 1981. By its definition, the soft computing is just known as an integration of a number of fields including the fuzzy logic, the neuro-computing techniques, the evolutionary procedures, the genetic computing and the statistical computing algorithms. This field leads to provide the combination of methods that can be used to represent a set of complex systems in modeling of which is impossible or very hard by applying computational regulations of pure mathematics and hard logic to them, whilst the applicable simulations and practical implementations may be conducted by using the soft computing techniques.

\footnotetext{
${ }^{1}$ Lotfali Askarzadeh (Baku 1920), Known as Lotfalizadeh or Lotfali A. Zadeh, Fuzzy Logic Founder and Professor of the University of Berkeley in California.
}

(- The Author(s) 2017. This article is distributed under the terms of the Creative Commons Attribution 4.0 International License (http://creativecommons.org/licenses/by/4.0/), which permits unrestricted use, distribution, and reproduction in any medium, provided you give appropriate credit to the original author(s) and the source, provide a link to the Creative Commons license, and indicate if changes were made. 
The key advantage of the soft computing is given, in its behavior, to present toward uncertain, approximate and relative systems. It leads to human-like behaviors, which are highly generalized. The development procedure of the boundaries of the soft computing field is now tabulated in Table 1.

Unlike hard computing, the soft computing is well-flexible against imprecision, approximation and partial truth, as well. The importance of using the soft computing techniques is generally clarified, as long as non-linear systems and also complex physical structures can all be modeled more precisely and flexibly, at a lower cost, and, in a shorter period of time, so they match with elite human decisions, in a high correctness percentage. The only noteworthy matter is that the soft computing is not precisely given as a concoction, mixture or combination, while it is considered to be a type of cooperation in which each member moves toward the desirable objective, in its unique way. The main principle in the soft computing is complementation, not competition. Therefore, the above-referenced soft computing is considered to be a foundation emerging in perceptual intelligence [1-3].

\section{The fuzzy-based approach and the neural networks}

Regarding the fuzzy-based approach, it should be noted that there are many types of fuzzy-based knowledge methods, in the real world, which indicate vague, imprecise, incorrect, uncertain and obscure behaviors, as well. Computing systems that are based on the classical theory of permutationor two-valued (binary) logic are not able to answer all the questions, which human can answer in general. In many cases, they produce numerous errors even if they have answers. Although, the assumption that the machine operates similarly to human is ideal, it is reasonable to expect the proposed system to realize the significant relationships in a problem (with an acceptable margin of error). It is quite clear that the behavior toward an uncertain problem should be flexible and therefore the fuzzy-based approach is applied to deal with such problems [4].

In this theory, the membership is specified through function $\mu(x)$ in the set, while $x$ indicates a specific member and the same $\mu(x)$ is a fuzzy-based function, which determines the membership degree of $x$, in the respective set. It is to note that its value is between zero and one.

$$
\tilde{A}=\left\{\left(x, \mu_{A}(x)\right) \mid x \in X\right\}
$$

The most intriguing application of fuzzy-based approach is the interpretation that this science provides for the structure of decisions made by smart beings and human intelligence above all. This logic well indicates why binary logic of classic mathematics is not able to explain and describe imprecise concepts, such as heat and cold, which constitute the basis of many smart decisions.

Table 1 Development of the soft computing boundaries [2]

\begin{tabular}{llr}
\hline Methods & Presenters & Years \\
\hline Neural network & McCulloch & 1943 \\
Evolutionary computing & Rechenberg & 1960 \\
Fuzzy logic & Zadeh & 1965 \\
Soft computing & Zadeh & 1981 \\
\hline
\end{tabular}


Regarding the neural networks, its development has started for almost 60 years ago. This science is based on imitating the brain performance in recreating the smart reasoning by computer. This imitation, however, is not the recreation of biological details or nerve cells. It is the exploitation of logical methods of brain performance, and the main principle is transferring these concepts in organization by special software applications. As computing speed increased in computers, the tendency to use neural networks in solving different problems is now increasing. Although common computing methods have advantages, the nature of their phase-to-phase and non-parallel processing is an obstacle to parallel processes pertaining to neural networks [5]. The artificial neuron is designed in order to imitate the first-order specifications of biological nerve cell. Naturally, a group of inputs is applied and each one represents the output of another one. Each input is multiplied by its corresponding weight, which represents the connection power. All these weighted inputs are summed, in order to determine the nerve stimulation level. Now, Fig. 1 indicates the neuron performance, mathematically.

A group of inputs which are shown as $x_{k}, k=1, \ldots, K$ are applied to the neuron. These inputs, which are totally considered as a vector resemble the signals are sent to the synapses of the nerve cell. Before being applied to summation unit, shown with a $\Sigma$, each signal is multiplied by its corresponding weight that represents the power of a biologically synaptic single connection. The summation unit that is slightly similar to the body of biological cell sums all weighted inputs algebraically and produces the output, which is here shown by $n$ representing $N E T$. This procedure may briefly be stated through a vector symbol as is $N E T=X W$.

\section{The particle swarm optimization algorithm}

The particle swarm optimization (PSO) algorithm is a method which is first recommended by Kennedy and Eberhart in 1995 [7]. It is based on the consideration of a simplified social model. The specifications of particles, which seek supply (target) in swarm, resulted in using it as a model to achieve optimized answers. In the usual implementation of this algorithm, the first position of the particle is determined, haphazardly. Using a series of assumptions and definitions, this natural model can be presented in the form of mathematic definitions. To establish a logical correspondence, a number of data inputs, which are called particles are assumed to be placed in a search-space in which each particle can consider a candidate for the solution. At first, each particle is placed in a random position and then it flies in a multi-dimensional space. The position of each particle toward itself and toward particles existing, in its proximity, is adjusted in each repetition (search). In these relocations, it is to note that each one of particles stores the

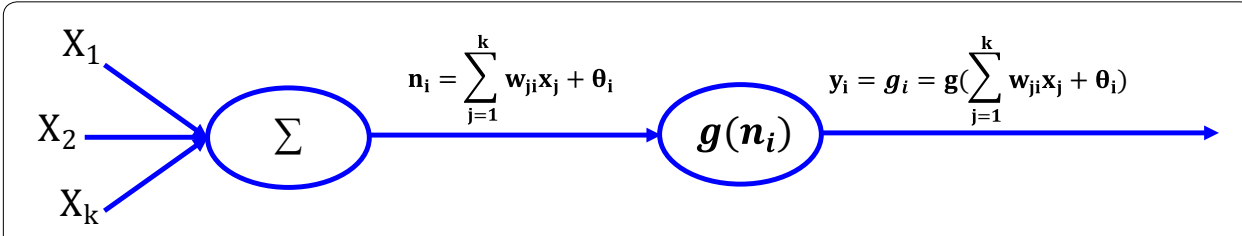

Fig. 1 The neuron performance in mathematic language [6] 
best position that is called pbest. It lets the particle select the best solution (fitness) for the local answers to the problem. Also, the best general position of particles is searched and tracked. It is called gbest. The above-referenced PSO algorithm adjusts the position each particle compared with the best value of pbest, which has been experienced. Also, the value of gbest is iteratively updated in accordance with the best answer to the problem, as its flowchart is briefly shown in Fig. 2.

\section{Reviewing the previous activities}

Controlling a complex process system such as the robot systems, power systems, communication channels and other similar ones benefit from fuzzy-based modulation and also the artificial neural networks most of times even when there is no previous knowledge of dynamic behavior of system's environment. An analysis of such the systems that

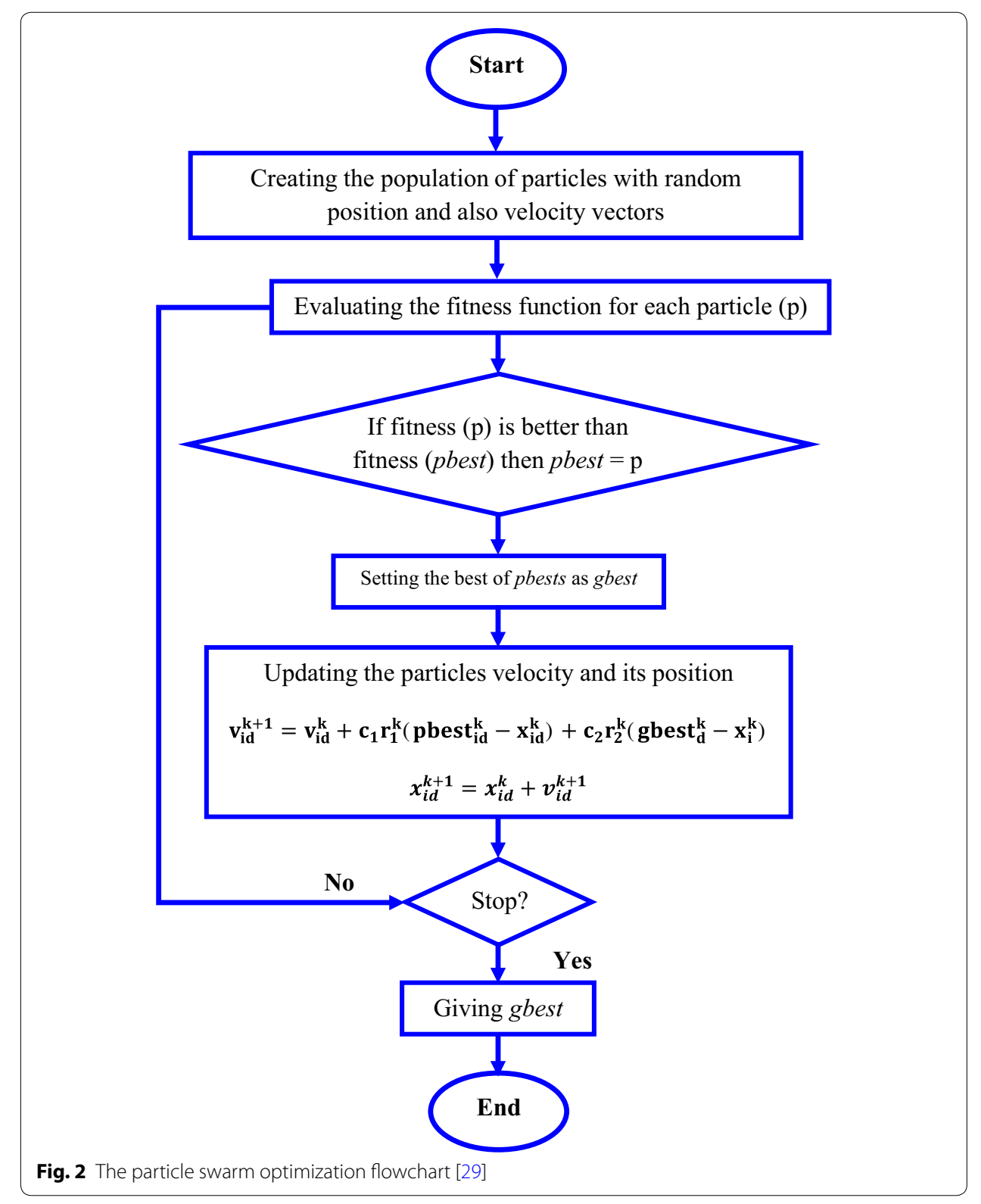


are controlled by the above-mentioned methods is addressed [8]. Robot control is related to the non-linear with time-varying nested combinations. Therefore, a precisely mathematic model cannot always be defined for its behavior and common techniques cannot also deal with the control systems of above-mentioned instruments. The main problems bring to attention, while controlling smart robots can sufficiently be considered $[9,10]$. Lack of timely performance, parameters with non-uniform distributions, non-minimal fuzzy-based behavior as well as hard connections and combinations are among the problems discussed. Due to these problems, the results of many relevant studies confirm that the matter of using the fuzzy-based approach by combining it with the neural networks can lead to a lot of improvement in controlling such systems [11]. Before that and in 1994, there is a study to investigate the impact of using the systems based on determining regulations [12]. An instance of such robots is here shown in Fig. 3.

The results of applying the soft computing methods in this field are so improving that several studies are presented in the form of authentic investigations. Now, it is considered to be one of the up-to-date interdisciplinary subjects to majors such as control, electronics, computer, mechanics and mechatronics. For instance, the effects of the fuzzy-based concepts can be discussed in the area of control systems of smart robots. The neural network is used to design the control system of a flexible robot system. The research can realize the artificial neural networks in the above-referenced system under control, in order to cope with the extent of a robot's movement, in a very high precision. As the technology of manufacturing instruments with high processing capabilities marches on, it is possible to apply both algorithms. The presence of the fuzzy-based approach, which increases the flexibility power and tolerance of uncertainty along with the neural networks advantages in terms of learning leads to the fact that the theory of concurrent becomes more powerful and the necessary incentive can be created for the studies of consistent with this idea. The research deals with the hybrid neural-fuzzybased technique in the area of flexible connections control approaches.

A description of neural-fuzzy-based control technique is presented, as the flexible control approach [9]. In the method presented, the weight factors that fuzzy-based control approach is given by the neural network with recursive nature are considered, while the same control approach operates without having any knowledge of control system's structure. A structure having four neural networks is introduced as the control system

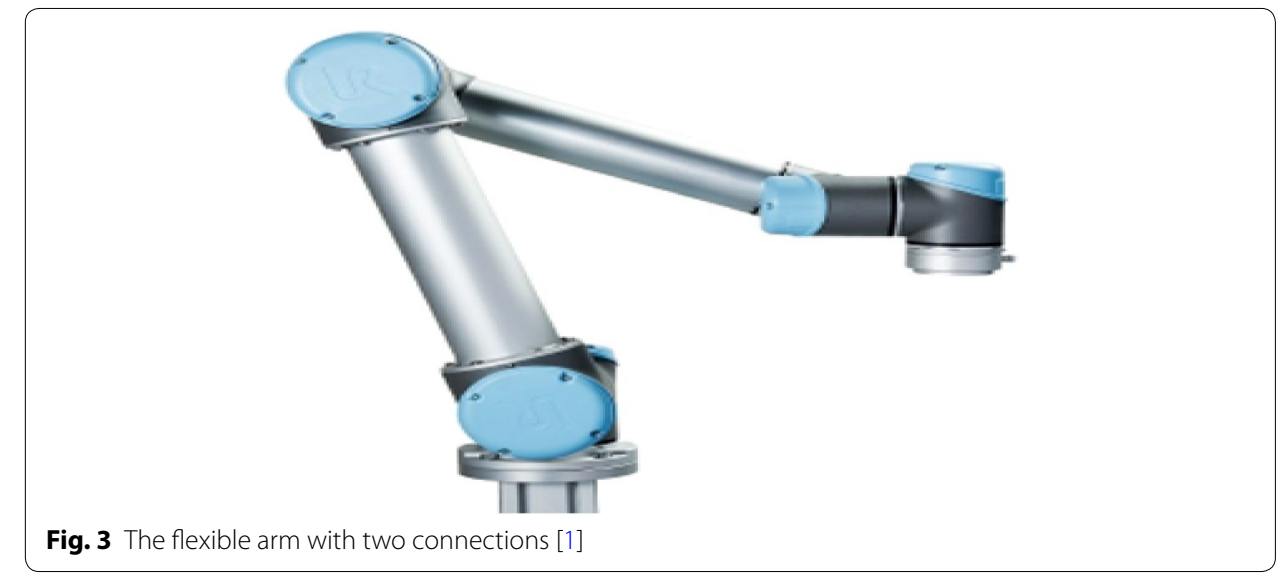


of tracking the position of a connection target with high flexibility. Its structuring is as follows: two neural networks are as the learning section of recursive errors, in order to educate the inverted dynamic correspondence to redefine the output of target position, the third neural network participates in the controlling operation with target function by learning the steepest decent technique and the fourth neural network which consists of two neural networks is applied to implement the online learning section and the appropriate output feedback (on the implementation of a system with minimal fuzzy-based behavior) [13-17].

Researchers named Lin and Lewis developed the fuzzy-based approach and applied to the singular perturbation method for controlling robot flexible arm system. Using this technique, two sub-systems of fast and slow have been created, where with these two sub-systems, automatic damp behavior and path tracking have been implemented [14]. A neural-fuzzy-based structure is proposed to control the target position of a flexible connection controlling the robot [15]. The factor of fuzzy-based control approach is in fact adjusted through a neural network, which is trained by the error post-distribution method. In the method proposed, a fuzzy-based control approach upon the inverted dynamic behavior is used so that tracking and refraction can be achieved under the control of such a system [19]. Moreover, the fuzzy-based in association with the neural networks are proposed to develop a self-organizing neural-fuzzy-based control approach, in order to deal with the tracking of the target position regarding the controlling system. The fuzzy-based regulations are determined and optimized in the control process concerning the method proposed. And the process of changing the membership values is conducted by applying the online neural network [19-29].

\section{The recommended control system}

Integral and derivation operands are frequently used in controlling the smart robot. These operands, which are represented as the traditional methods in the area of control systems can be considered as the proportional-integral-derivative (PID) that is maintained in non-classic methods in terms of the general structure. Unlike fuzzy-based control approaches, a great set of input-output variables are used. It is because each section is fed by more than one input in systems with high connectivity. Therefore, a multivariable fuzzy-based control approach can be designed to control such situation of this system. The usual procedure of designing these systems is in a way that the general system is divided into some sub-systems, which are multiple inputs single output (MISO). Each of these sub-systems goes through the path from input to output in a parallel and coordinated manner [14-21]. In the proposed method, the minimal values of membership functions are first chosen in accordance with three referenced values and then the maximizing value is applied. The multiple inputs multiple outputs (MIMO) structure regarding the fuzzy-based control approach is shown in Fig. 4 for the flexible control of multi-connectional hard connections.

Two consecutive links $(i \mathcal{E} i+1)$ are assumed in this figure. The fuzzy logic control (FLC) of input $i$ includes the angle error connection $\left(e_{i}=\theta_{d i}-\theta_{i}\right)$ and also the acceleration signal at the tip of $a_{t i}$. The scale factor is related to the tracking error, the acceleration at tip and input torque for FLC of input $i$. It is respectively equal to $k_{p i}, k_{a i}$ and $k_{t \mathrm{i}}$. Now, the FLC of input $i+1$ also follows the procedure of input $i$ and controls its 


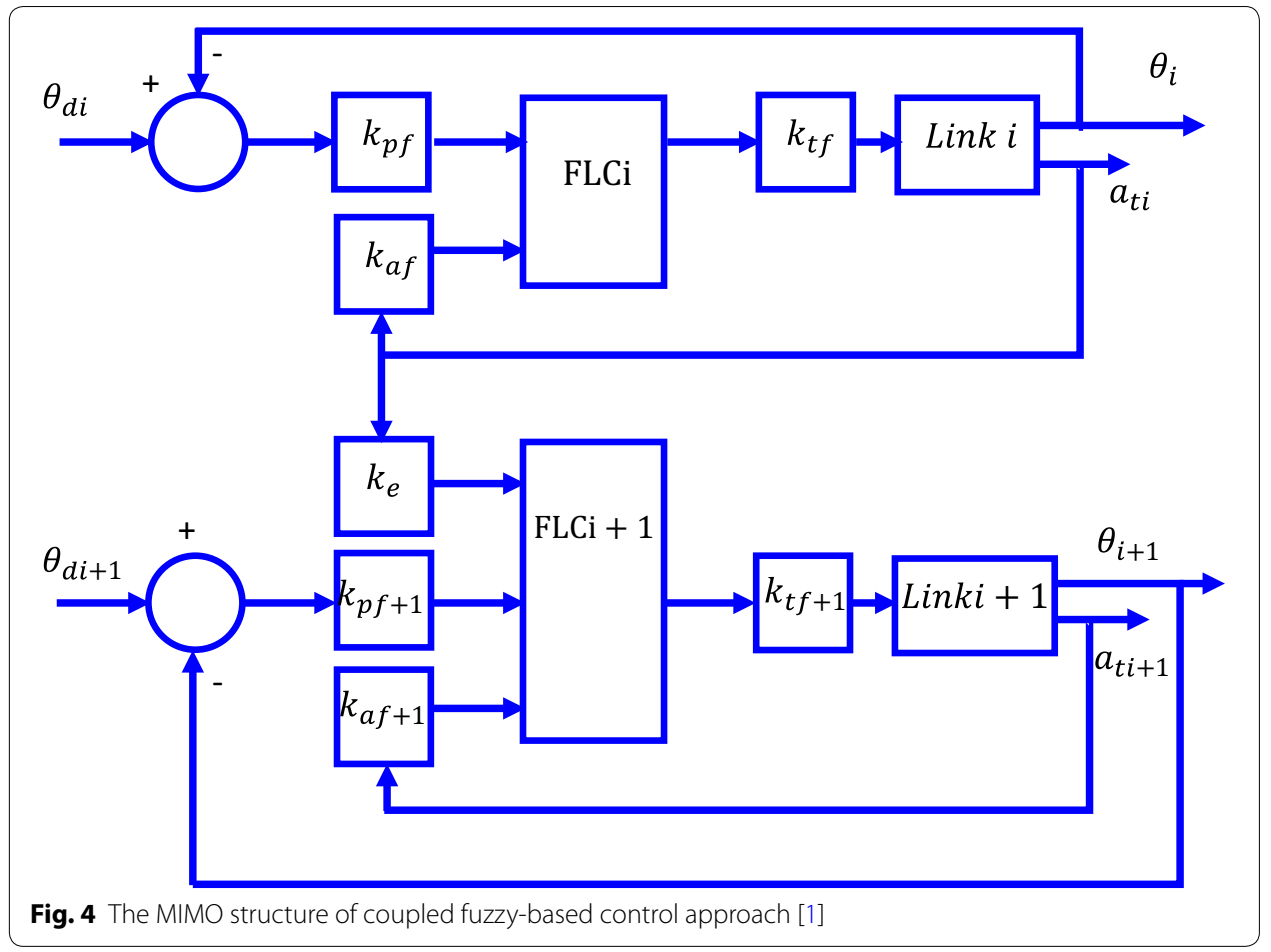

relevant output. It is recommended that scale factors to be normalized in the period of $[-1,1]$, so the computing process becomes easier.

\section{The radial based function neural network training}

In the radial based function neural network (RBFNN) training, the center and the width of the Gaussian function is kept constant, in order to generate a fixed mapping between the input and the hidden layer. The above-referenced RBFNN output weights can be determined by traditional training algorithms such as the least mean square (LME), the normalized least mean square (NLMS), the evolutionary algorithms (EA) and the PSO. The main advantage of the RBFNN is its structure rapid simplicity and high estimation [22]. The structure of this combination consists of three layers entitled (1) the input layer, which includes input nodes, (2) the hidden layer in which each neuron uses the radial based function as its activity node and (3) the output layer, which transfers a linear combination of summed values of output layer activities to the output. The proposed neural network complexity is now illustrated in Fig. 5.

\section{Determining neural networks factors by using PSO algorithm}

The PSO algorithm is a method which is based on the simplified social model. The specifications of particles that seek target in swarms, is given as the model to achieve optimized answers. This procedure is now described by the following

$$
\left\{\begin{array}{l}
V_{i}(t+1)=w V_{i}(t)+c_{1} r_{1}\left[P_{i}(t)-X_{i}(t)\right]+c_{2} r_{2}\left[P_{g}(t)-X_{i}(t)\right] \\
X_{i}(t+1)=X_{i}(t)+V_{i}(t+1)
\end{array}\right.
$$

Here, $X_{i}=\left\{x_{i 1}, \ldots, x_{i n}\right\}^{T} \in \mathrm{S}$ and $V_{i}=\left\{v_{i 1}, \ldots, v_{i n}\right\}^{T} \in \mathrm{S}$ are taken, while $\mathrm{S} \subset \Re$ is the search-space. Here, $X_{i}(t+1)$ is the next position of particle that is updated in accordance 


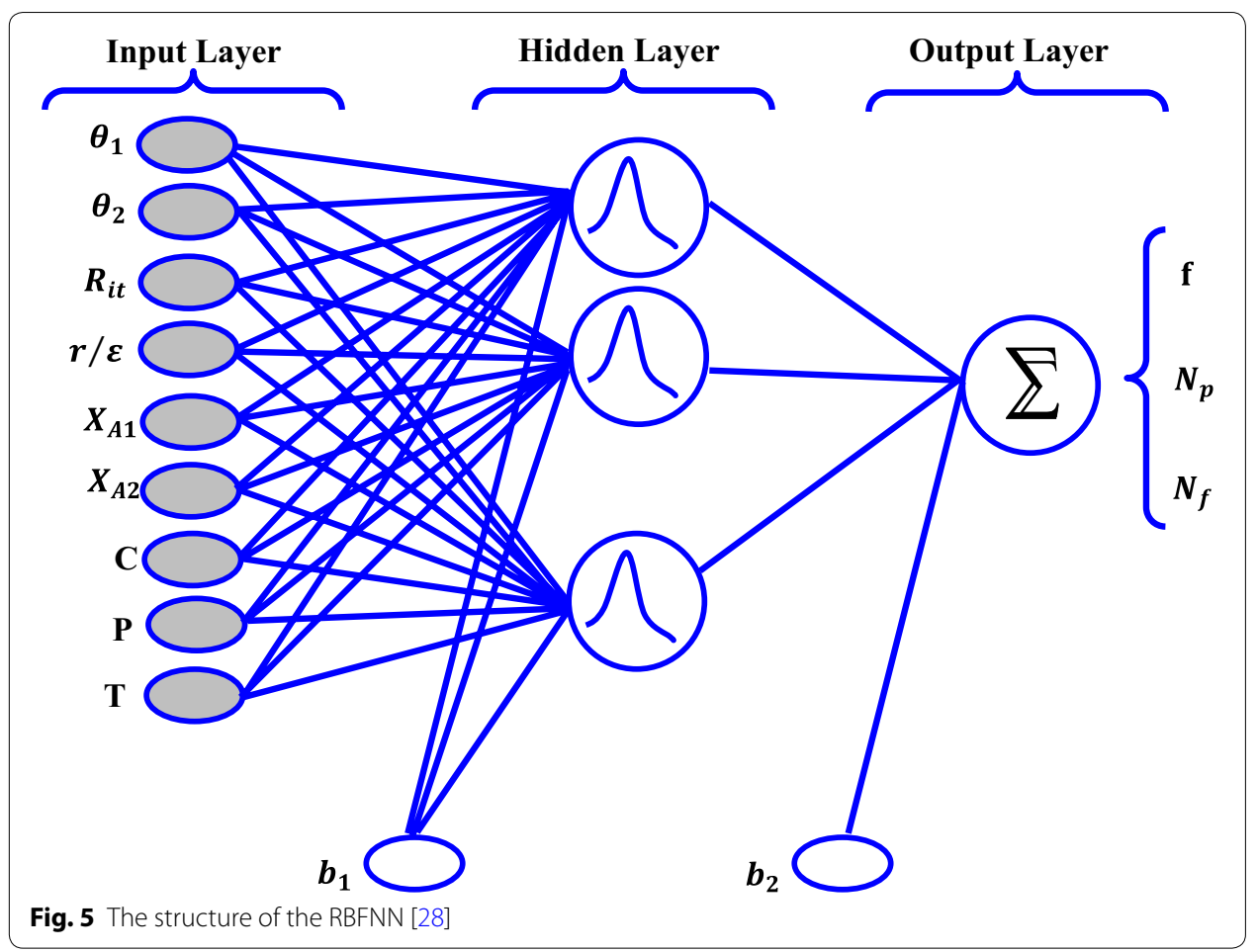

with the value of $V_{i}(t+1)$. In fact, this procedure is applied to determine $W_{i}(t+1)$ from $W_{i}(t)$ and therefore the whole of weight factors can efficiently be updated.

\section{The configuration of the proposed fuzzy-based approach}

As it has been pointed out, designing the multi-variable control approach for a flexible arm with some links, which are in the form of soft and hard connections should benefit from the fuzzy-based approach, in order to have a desirable functionality. In fact, control systems are considered to be in the form of the MIMO. However, the implementation of such systems is difficult and its computations are time-consuming.

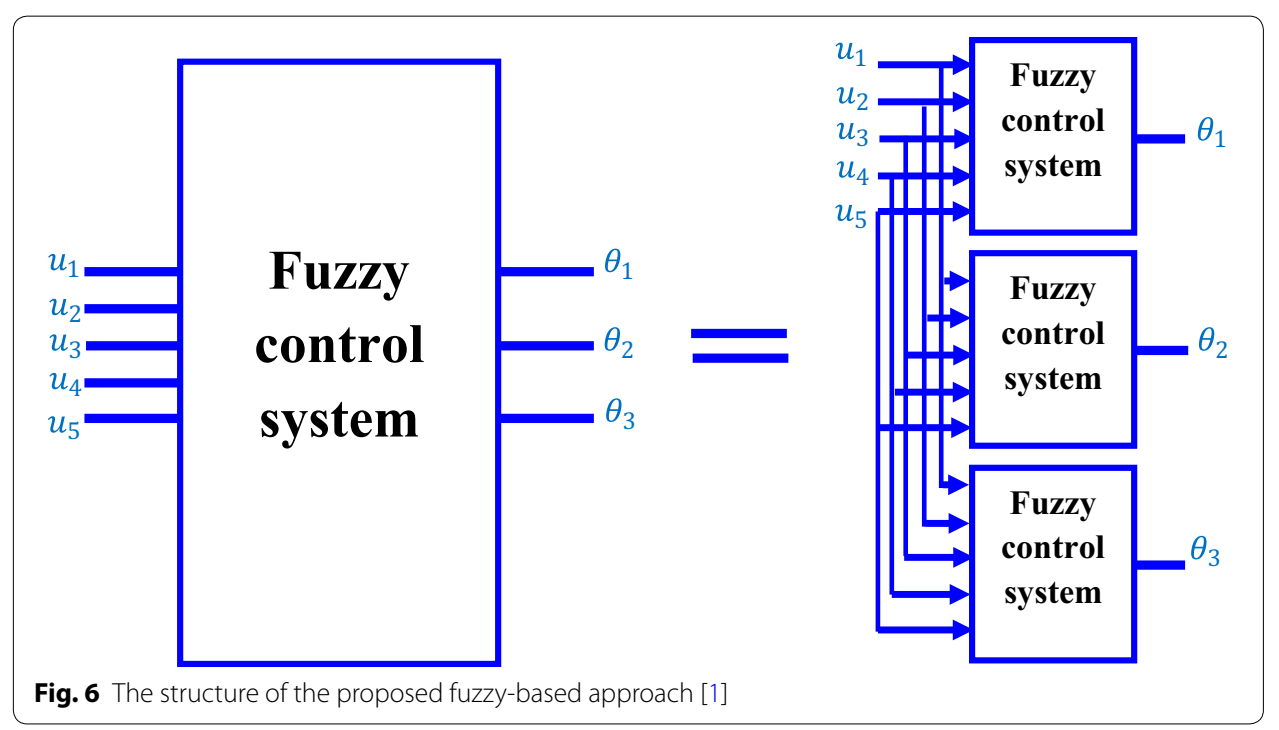


Table 2 Types of movements from fuzzy-based regulations and claw position [1]

\begin{tabular}{cllllllll}
\hline & $\delta \boldsymbol{\theta}_{\mathbf{1}}$ & & & & & & & \\
\hline$A_{i}$ & & $N B$ & $N M$ & $N S$ & $Z$ & $P S$ & $P M$ & $P B$ \\
& $N B$ & $P B$ & $P M$ & $P S$ & $Z$ & $N S$ & $N M$ & $N B$ \\
& $N M$ & $P B$ & $P M$ & $P S$ & $Z$ & $N S$ & $N M$ & $N B$ \\
& $N S$ & $P B$ & $P M$ & $P S$ & $Z$ & $N S$ & $N M$ & $N B$ \\
$Z$ & $Z$ & $Z$ & $Z$ & $Z$ & $Z$ & $Z$ & $Z$ \\
& $P S$ & $N B$ & $N M$ & $N S$ & $Z$ & $P S$ & $P M$ & $P B$ \\
& $P M$ & $N B$ & $N M$ & $N S$ & $Z$ & $P S$ & $P M$ & $P B$ \\
& $P B$ & $N B$ & $N M$ & $N S$ & $Z$ & $P S$ & $P M$ & $P B$ \\
\hline
\end{tabular}

Therefore, the structure of some MISO sets is of the proposed method, as is now shown in Fig. 6.

Also, a regulation is required for the two-connection arm so that the robot's claw can be placed in the right position. The applied regulation is given as follows

If $\left(\mathrm{A}_{1}\right.$ is $\left.\mathrm{NM}\right)$ and $\left(\delta P_{x}\right.$ is $\left.\mathrm{PS}\right)$ Then $\delta \theta_{1}$ is NS

Accordingly, the following results are first obtained as tabulated in Table 2.

The definitions of each movement via membership function are then indicated in Table 3.

The proposed neural fuzzy-based network is finally illustrated in Fig. 7.

The proposed structure is based on the RBFNN and the Sugeno-type of the fuzzybased approach. It should be noted that the same fuzzy-based approach is similar to the popular Mamdani-type method, except that for the output membership functions, there exists a singleton spike instead of the distributed fuzzy-based sets. The fuzzy-based network outlined is comprised by five layers. Each group in first layer is in the form of the adaptive node, which its function is determined as follows

$$
O_{1}^{i}=\mu_{A i}(T)
$$

$\mathrm{T}$ is the first input vector and $\mu$ is the membership function for the real input. The second layer involves the fixed nodes, while the output of each node is produced by two membership functions:

$$
O_{2}^{i}=W_{1}=\mu_{A i}(T) \mu_{B i}(P)
$$

The third layer includes the fixed node with produced by the normalizing function as given by

$$
O_{3}^{i}=\bar{W}_{i}=\frac{W_{1}}{W_{1}+W_{2}} i=1,2
$$

Table 3 The definitions of robot's claw movement

\begin{tabular}{ll}
\hline Membership function & Definitions \\
\hline PB & Positive big \\
PM & Positive medium \\
PS & Positive small \\
$Z$ & Zero \\
NS & Negative small \\
NM & Negative medium \\
NB & Negative big \\
\hline
\end{tabular}




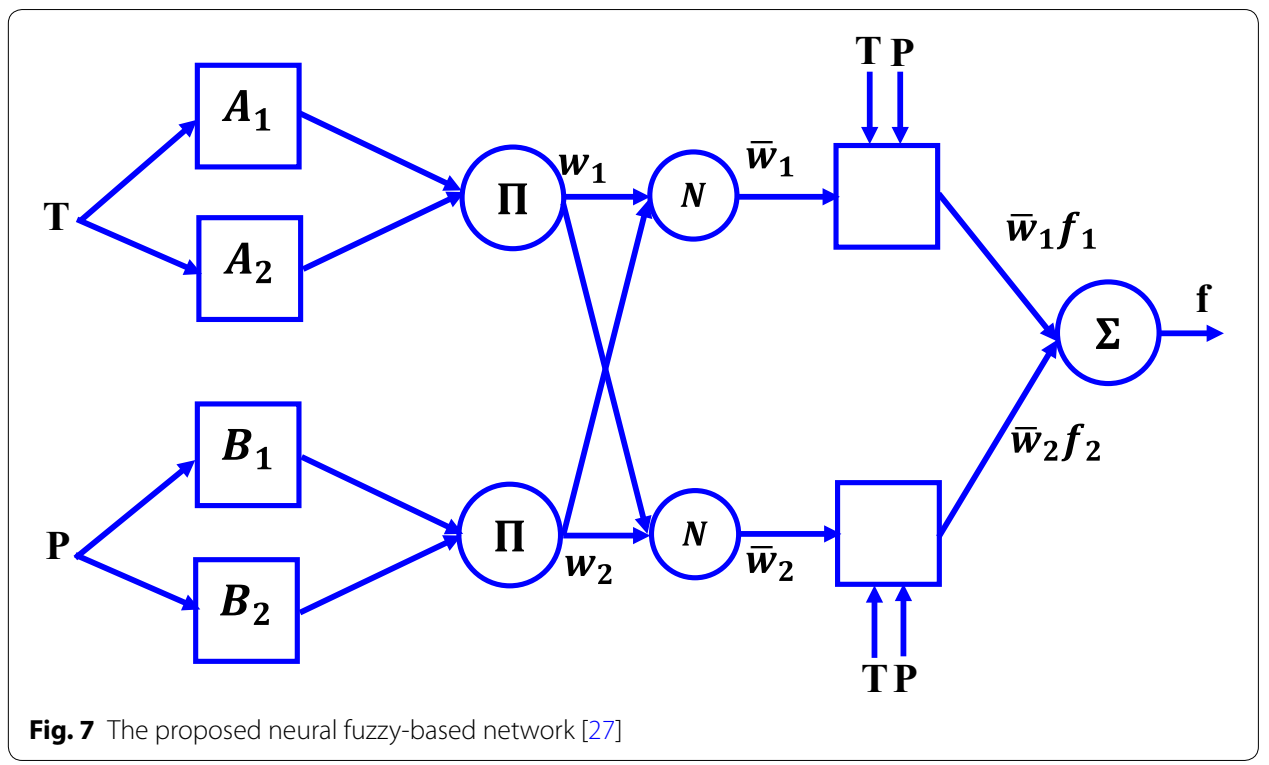

The fourth layer as is now outlined by the square shape is produced though calculating the consequent parameters and the third layer's output, so that

$$
O_{4}^{i}=\bar{W}_{i}\left(P_{i} T+q_{i} P+r_{i}\right)
$$

Finally, the fifth layer as is also outlined by the circle shape calculates the input signals. The structure is actually taken for the Sugeno fuzzy-based model.

$$
O_{5,1}^{i}=\sum_{i} \bar{W}_{i} f_{i}
$$

For a normal structure, the network's parameters, especially its weight factors, are updated by reducing the gradients. However, here, the process is replaced by the PSO.

\section{The topology of hybridizing fuzzy-based neural network approach}

The integration of the fuzzy-based approach in association with the neural networks for controlling the robots is organized in the two general classes, in this research. First, the outcome has to be capable of learning the functions. In other words, before the fuzzybased rules are implemented, it makes some preprocesses for optimizing the data. Second, the fuzzy-based neural control approach upon the investigated topology is realized. Here, the hybridizing technique regarding the fuzzy-based neural network is considered to design a control approach. In order to reduce error and the time consumed, the PSO algorithm is applied to produce the weight factors and the Gaussian function's center. The network is in fact designed in five layers, where the corresponding outcomes are simulated through a flexible manner, in order to control the two arm's robotic movements so that this one of the circulation ring could be taught, as shown in Fig. 8.

\section{Simulation results}

The simulations are all carried out by considering the flexible arm system with two connections, in its general scheme, as indicated in Fig. 9. 


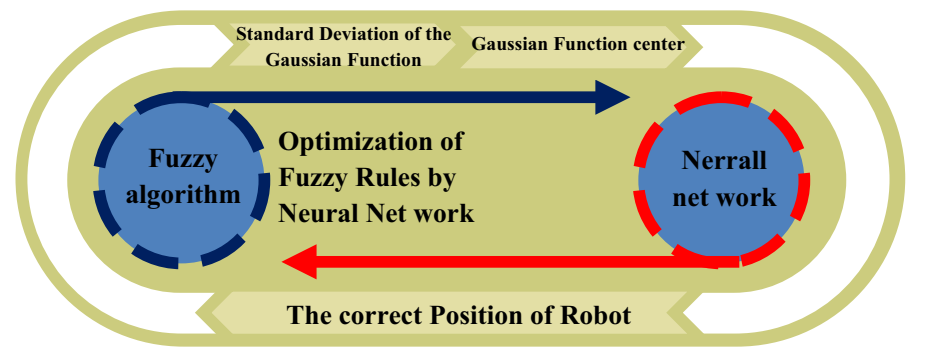

Fig. 8 The general structure of proposed radial based neural network

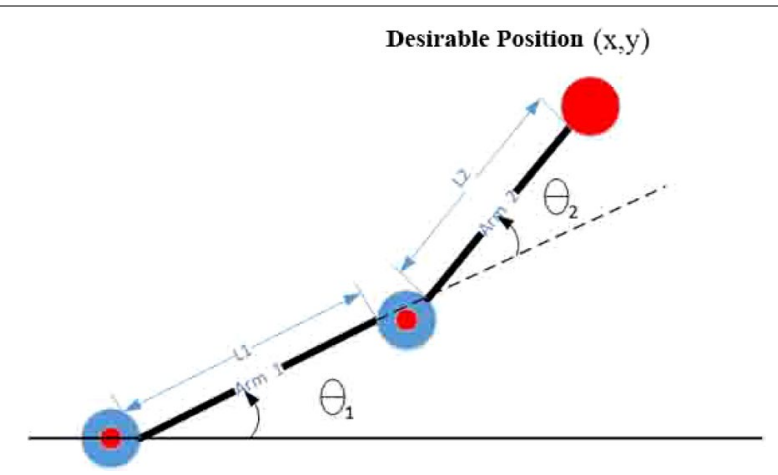

Fig. 9 The flexible arm system with two connections

Given the structure of the above-captioned flexible arm with two connections, a neural network learns to map the coordinate $(\mathrm{x}, \mathrm{y})$ on $\theta_{1}$ and $\theta_{2}$. The trained adaptive neurofuzzy inference system (ANFIS) is realized as one part of the control system, which is responsible for coping with the robot's arm. Knowing the desirable position of arm tip, the network is responsible for generating the optimal values of angles and applying the necessary force to the correct direction, in order to reach those angles so that it can gpuide the arm tip to the desirable position. The $\theta_{1}$ is taken as the angle between the first arm and the ground, while $\theta_{2}$ is also taken as the angle between the first arm and the second arm. For instance, the minimal length of the first arm is $11 \mathrm{~cm}$, where the length of the second arm is $12 \mathrm{~cm}$. Furthermore, the first arm is able to rotate from $0^{\circ}$ to $90^{\circ}$, where the second angle varies between $0^{\circ}$ and $180^{\circ}$, as Fig. 10 explains this matter of fact.

Now, it should be able to define the coordinates of $x$ and $y$ in the system. Therefore, there are

$$
\left\{\begin{array}{l}
X=L_{1} \cos \left(\theta_{1}\right)+L_{2} \cos \left(\theta_{1}+\theta_{2}\right) \\
Y=L_{1} \sin \left(\theta_{1}\right)+L_{2} \sin \left(\theta_{1}+\theta_{2}\right)
\end{array}\right.
$$

Now the sum of data can be generated:

$$
\left\{\begin{array}{l}
\text { data } 1=\left[X, Y, \theta_{1}\right] \\
\text { data2 }=\left[X, Y, \theta_{2}\right]
\end{array}\right.
$$

Here, the overall performance of claw in the positions, specified in the previous one can be illustrated in Fig. 11, as it is now clarified that robot claw is able to go through the path specified by training points. 


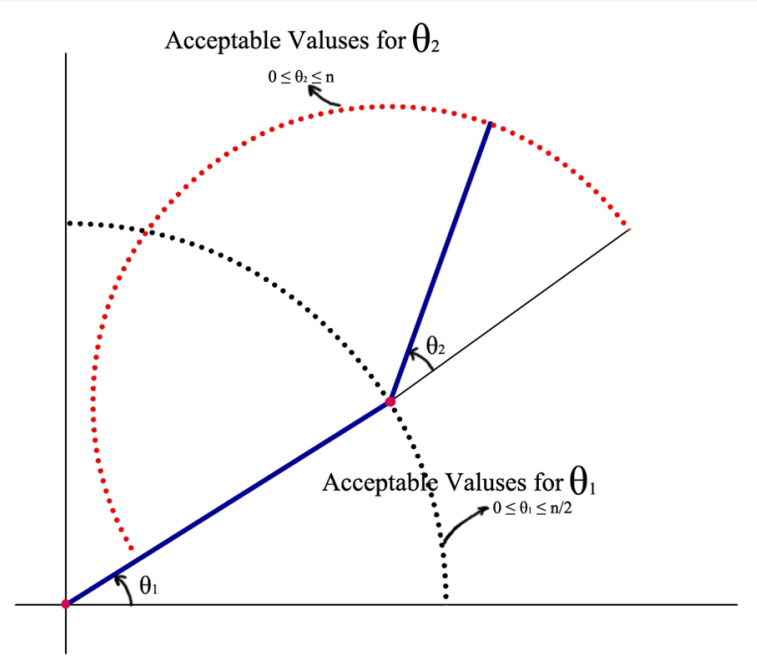

Fig. 10 Conditions considered for the rotation of robot arms system

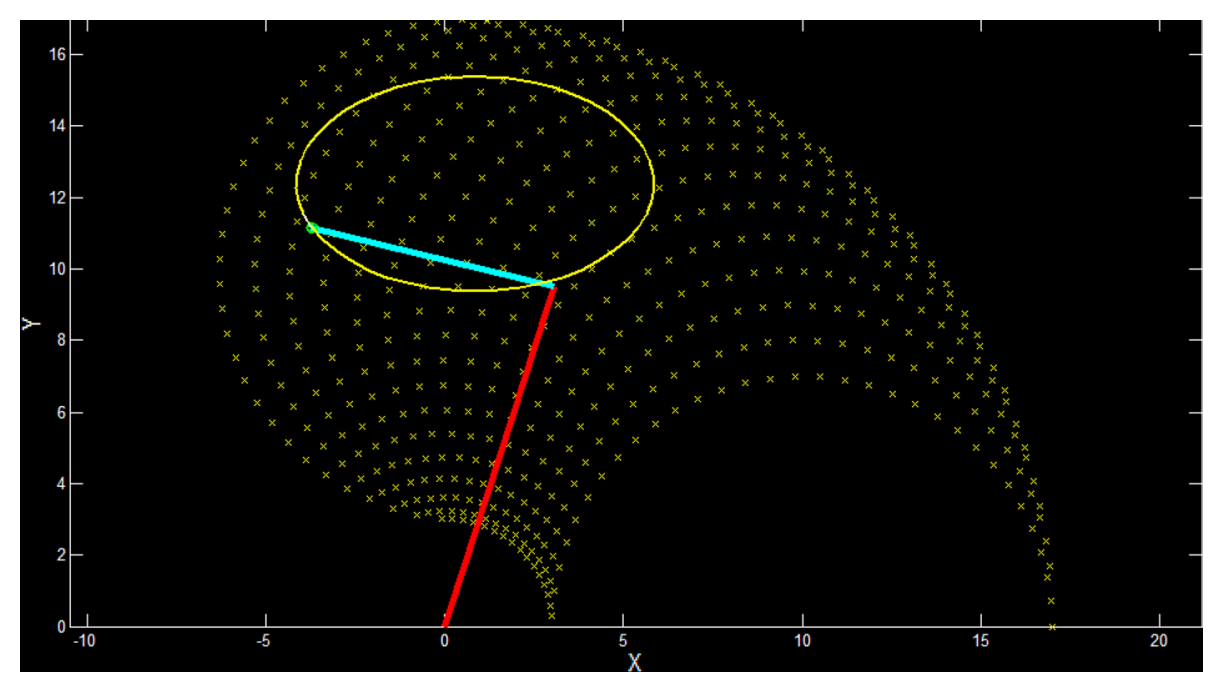

Fig. 11 Claw movement on a ring assumed by using the proposed network

Because of the fact that the fuzzy-based neural network approach is designed to enable the robot to be taught in spaces outside the region, the outcomes are also moving in the right direction, illustrated in Fig. 12.

It is to note that these results are in fact carried out by configuring the proposed fuzzybased neural network approach through the training of the RBFNN via the PSO algorithm, where the differences between real and target output for all training data can be apparent. Now, by extracting the parameters of the regression, the mean-square error (MSE), the root-mean-square error (RMSE) and changes in values of $\mu$ and $\sigma$ of the Gaussian functions regarding the RBFNN in training, the performance of the approach proposed here using the behavior of robot's claw along the assumed path can further be considered, as are all shown in Figs. 13, 14 and 15 in the scenarios 1, 2 and 3, respectively. 


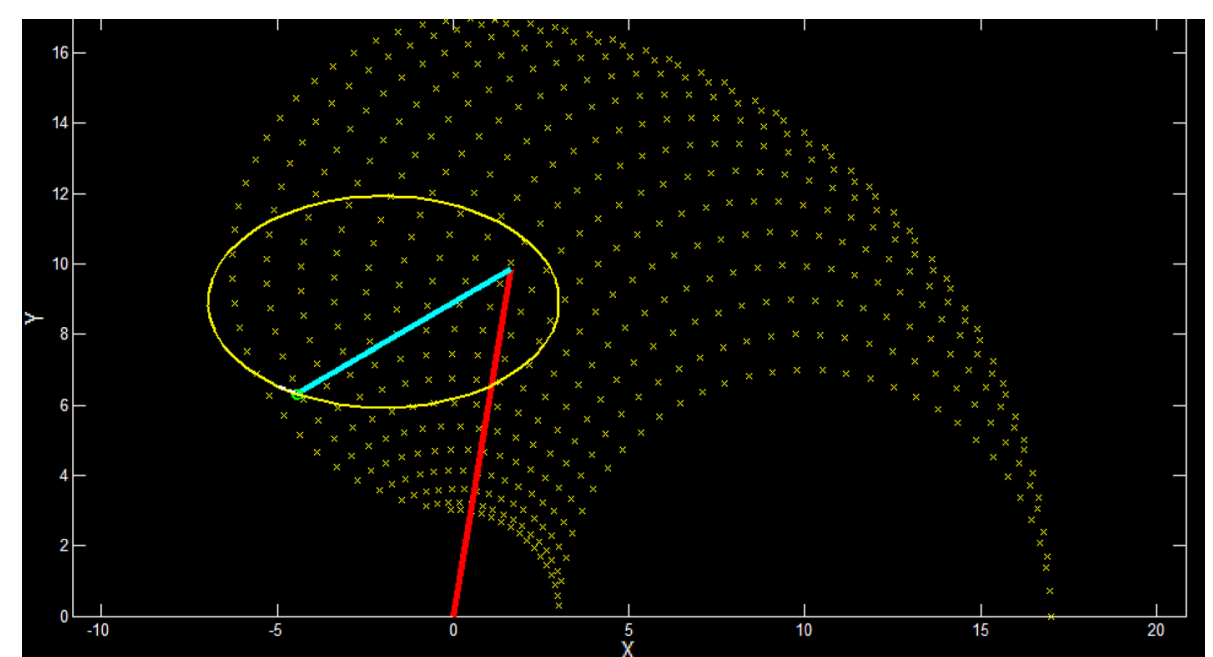

Fig. 12 Correcting the claw on the outside of the trained robot

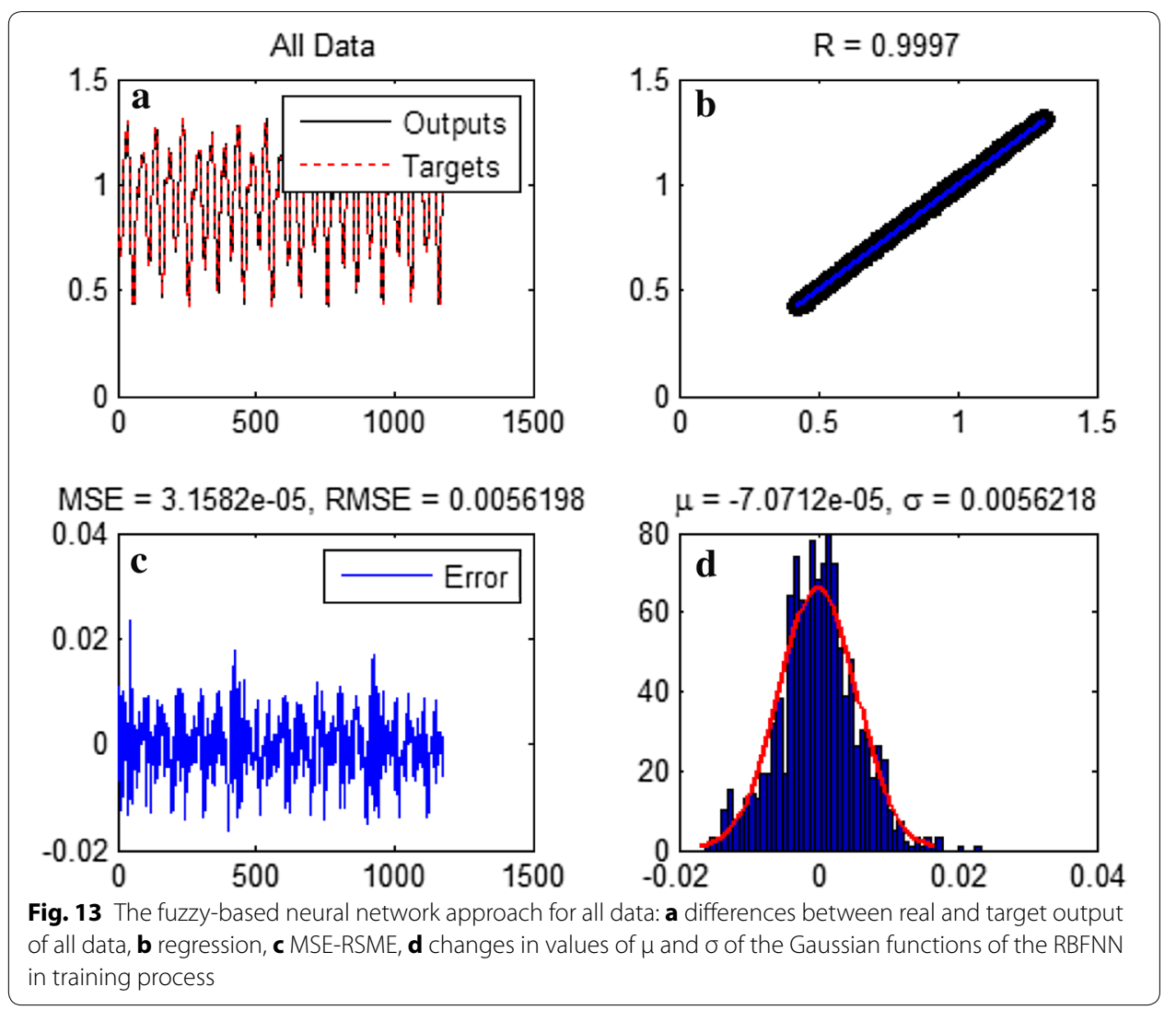

The algorithm is clear to be based on the social model and therefore it is important to consider the position of the particle including pbest and gbest, illustrated in Fig. 16.

Regarding the arm's stability in the different positions, the investigated outcomes are all clearly shown in Figs. 17 and 18, while the overall desirable performance of the system under control in a number of different scenarios are achieved. 


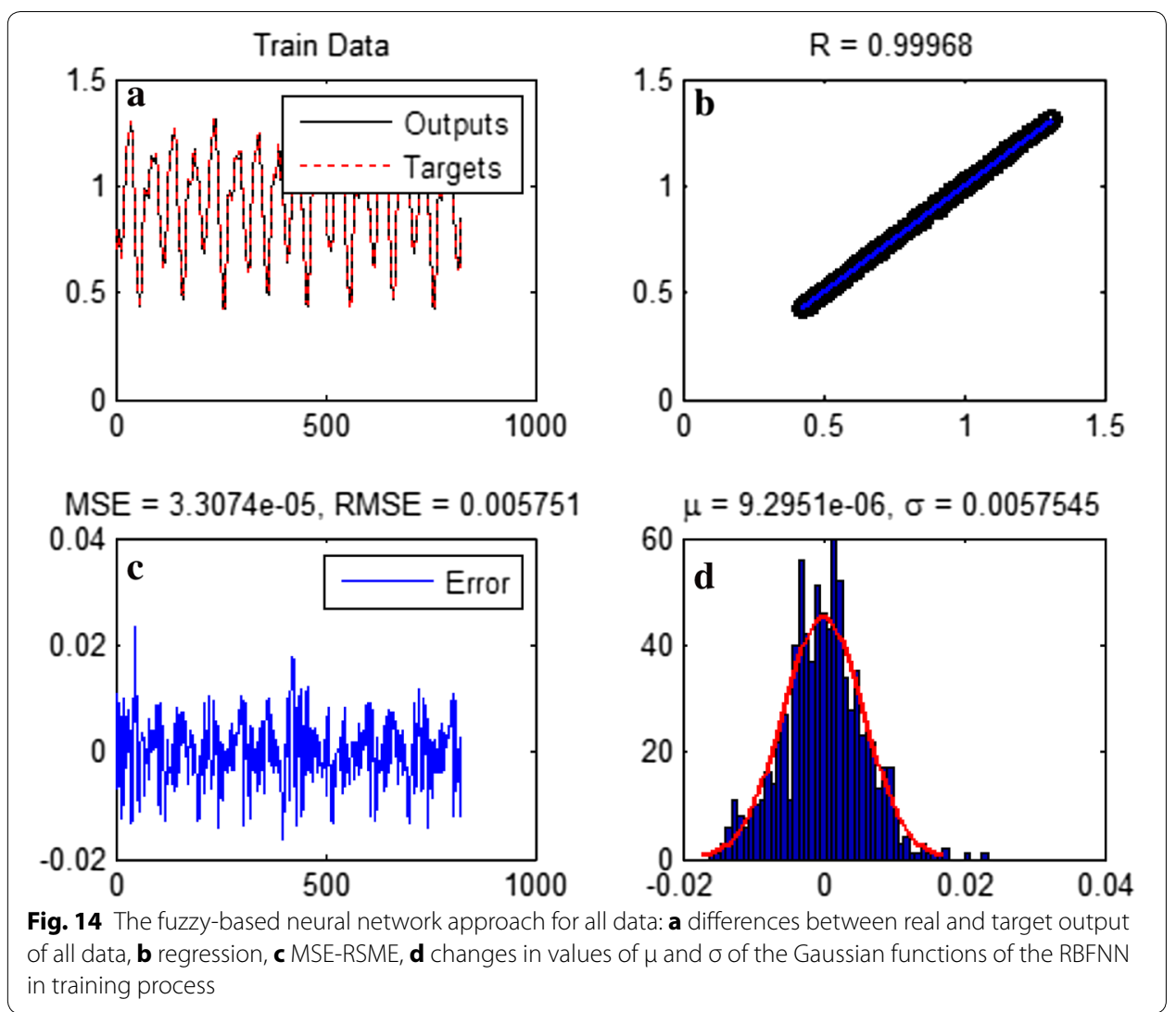

The final values of the MSE, the RMSE and the regression of the proposed fuzzy-based neural network approach are indicated in Table 4.

The considerable improvement of the proposed fuzzy-based neural network approach can be considered, as is now tabulated in Table 5, with reference to a number of the previous benchmarks, in this area, as well. In the two-dimensional input space with the twoconnection arms, having specific coordinates, the main problem is to find two involved angles. The first angle is the one between the first arm and the horizon, as long as the second angle is the one between the first arm and the second arm, respectively.

\section{Conclusion and its recommendation}

A hybridized idea of fuzzy-based neural network approach has been designed as the proposed control approach, in this investigation. In the approach realized here, the PSO algorithm is used to decrease error and time, in order to generate the neural network factors. The aforementioned network is in fact designed in five layers to control the movement of the robot system under control with two arms, in a completely flexible manner, in which this network can momentarily be trained by moving the rotation ring. By using the Sugeno-type of the fuzzy-based approach, circumstances have been provided for the robot claw to cover even the untrained areas. Although these areas are now limited, arm claw's ability is upgraded to move, efficiently. This ability is in fact 

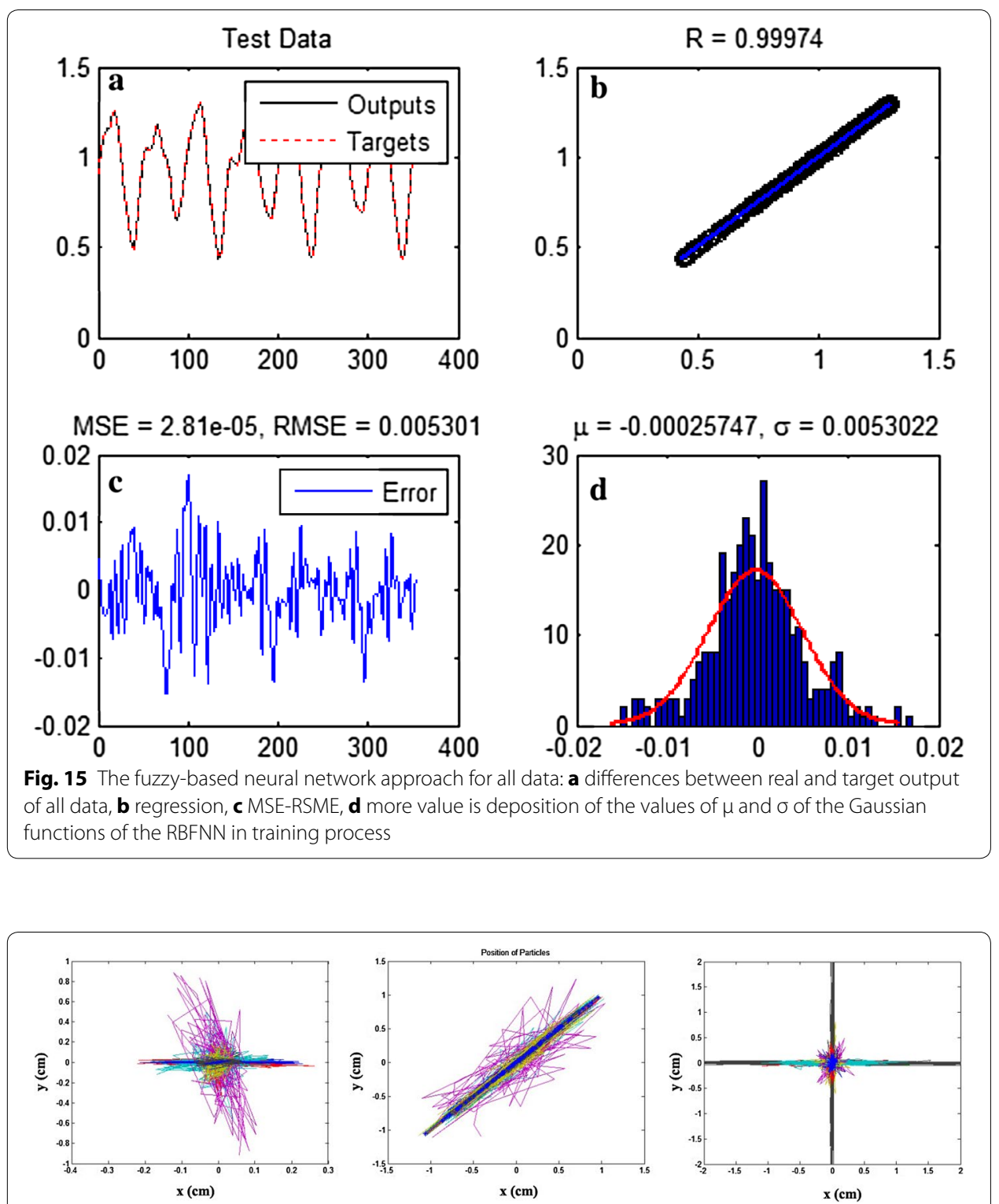

Fig. 16 The position determined by the PSO algorithm

due to the Sugeno-type of the fuzzy-based approach that is fewer than 150 regulations. Moreover, the arm claw is illustrated, in order to achieve the balanced state. This parameter, which is very important to consider in movement control systems, indicates the speed and precision of the claw, in the achieving balanced state. Furthermore, the investigated results can be improved by applying some changes to the internal structures of each fuzzy-based neural network framework. For instance, the neural networks such as the multilayer perceptron (MLP), the Kohonen and other ones can be used instead of the RBFNN. Also, the genetic algorithm (GA), the imperialistic competitive algorithm and other related ones may correspondingly be used instead of the PSO algorithm, in 


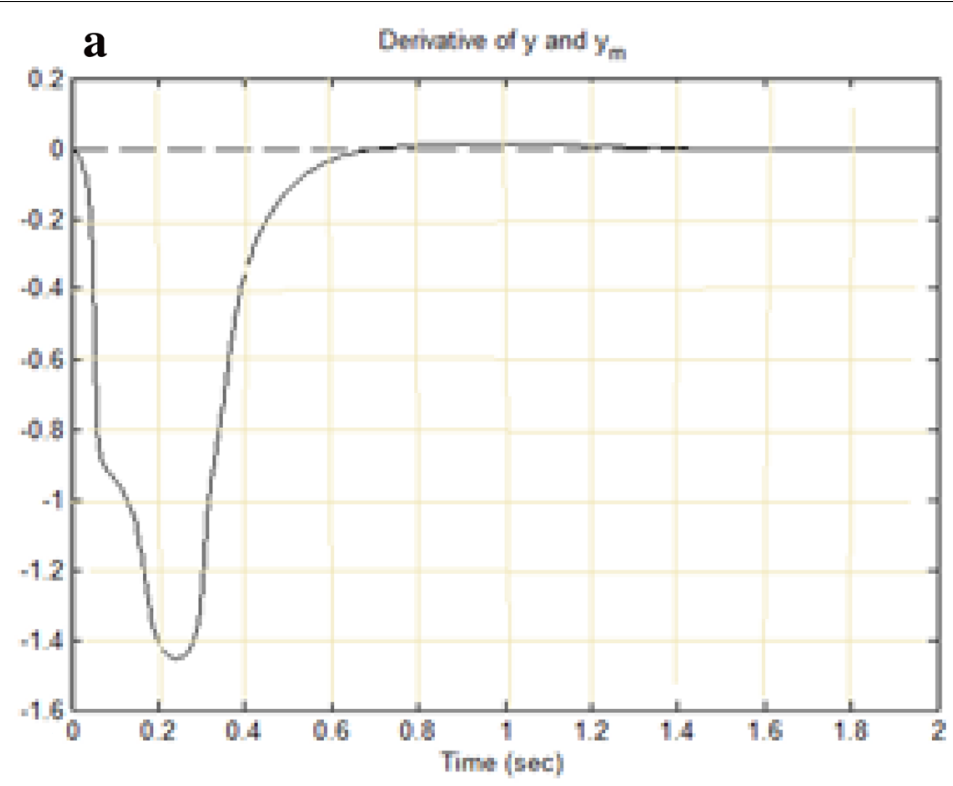

b Roll angle $y(t)$ (solid) and $y_{m}(t)$ (dashed)
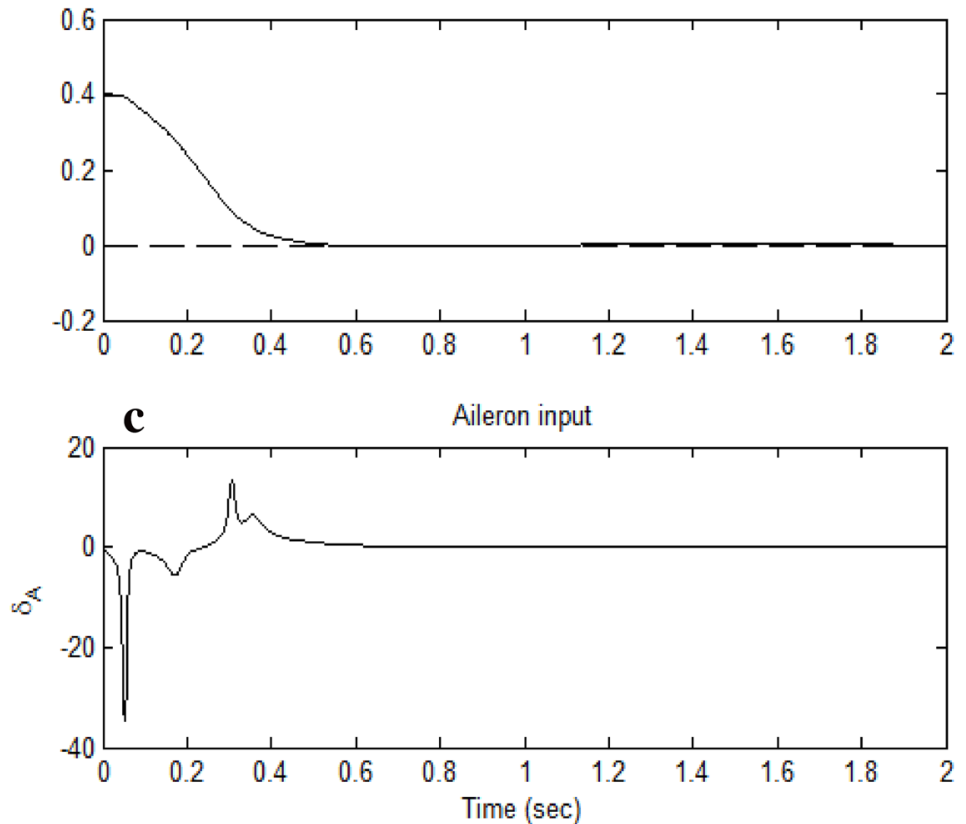

Fig. 17 a The shake robot arm to sustainability, $\mathbf{b}$ the stability of the robot, $\mathbf{c}$ the Arm's stability in one of the assumed states 


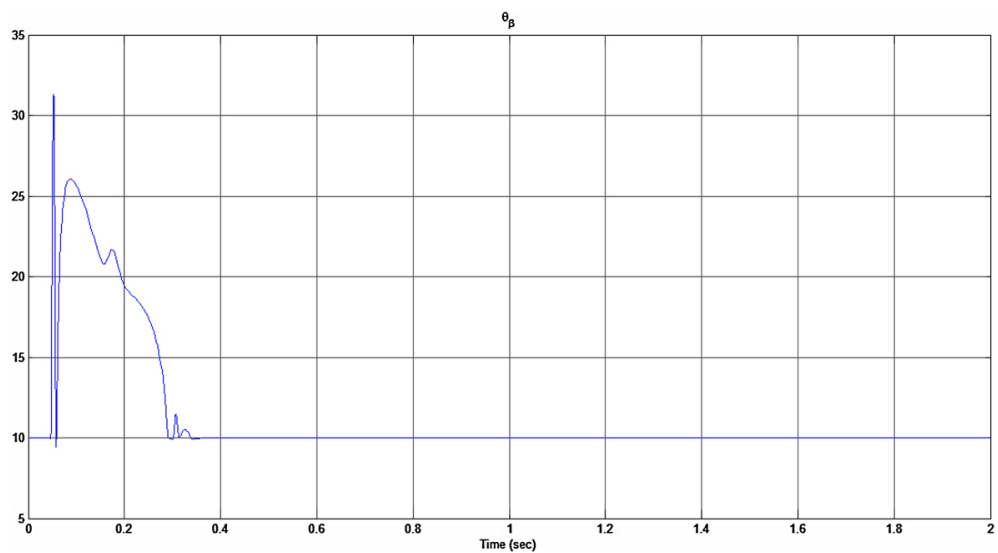

Fig. 18 The robots' arm slip befor reaching the balance

Table 4 The MSE and, the RMSE and the regression of the proposed fuzzy-based neural network approach

\begin{tabular}{llll}
\hline & Test data & Training data & All data \\
\hline Regression & 0.99687 & 0.99999 & 0.99999 \\
MSE & $281.25 \mathrm{e}-5$ & $9.9841 \mathrm{e}-5$ & $91.353 \mathrm{e}-5$ \\
RMSE & 0.053029 & 0.009992 & 0.030255 \\
\hline
\end{tabular}

Table 5 A number of the previous investigations with reference to proposed approach

\begin{tabular}{lll}
\hline Approach/benchmark & RMSE & MSE \\
\hline The proposed approach & 0.053029 & $281.25 \mathrm{e}-5$ \\
Mote approach [24] & - & $2.003 \mathrm{e}-2$ \\
Mohammad approach [25] & $44.67 \mathrm{e}-1$ & $6.6667 \mathrm{e}-2$ \\
Bazargan approach [26] & - & 0.0637 \\
\hline
\end{tabular}

order to determine the key parameters of the neural networks. It can be shown that each of these can cause a variation in the overall outcome of the aforementioned control approach.

Authors' contributions

AHM carried out the proposal of designing the method and its verification. EK carried out the initial procedure of designing the method and its simulations. Both authors read and approved the final manuscript.

Author details

${ }^{1}$ Department of Control Engineering, South Tehran Branch, Islamic Azad University (IAU), No. 209, North Iranshahr Street, P.O. Box 11365/4435, Tehran, Iran. ${ }^{2}$ Department of Control Engineering, Faculty of Electrical Engineering, South Tehran Branch, Islamic Azad University (IAU), No. 209, North Iranshahr Street, P.O. Box 11365/4435, Tehran, Iran.

\section{Competing interests}

The authors declare that they have no competing interests.

Received: 12 May 2015 Accepted: 16 January 2017

Published online: 27 January 2017 


\section{References}

1. Subudhi B, Morris AS (2009) Soft computing methods applied to the control of a flexible robot manipulator. Appl Soft Comput 9(1):149-158

2. Ahmad I (2013) Optimized intrusion detection mechanism using soft computing techniques. Telecommun Syst $52: 2187$

3. Mazinan AH (2015) High-precision full quaternion based finite-time cascade attitude control strategy considering a class of overactuated space systems. Human Centric Comput Inf Sci 5:27

4. Martínez R, Castillo O, Aguilar LT (2009) Optimization of interval type-2 fuzzy-based control approaches for a perturbed autonomous wheeled mobile robot using genetic algorithms. Inf Sci 179(13):2158-2174

5. R. Bill V. T. Jackson, translated by M. Alborzi (2007) An introduction to neural networks, third edition. Sharif University of Technology Press, Tehran, p 138

6. Huang Y, Lan Y, Thomson SJ, Fang A, Hoffmann WC, Lacey RE (2010) Development of soft computing and applications in agricultural and biological engineering. Comput Electron Agric 71(2):107-127

7. Kennedy J, Eberhart RC (1995) Particle swarm optimization. In: Proceedings of IEEE international conference on neural networks, Perth, p. 1942-8

8. Linkens DA, Nyongesa $\mathrm{HO}$ (1994) Learning systems in intelligent control: an appraisal of fuzzy-based -based, neural and genetic algorithm control applications. IEE Proc Control Theory Appl 143(3):367-386

9. Tian L, Collins C (2005) Adaptive neuro-fuzzy-based control of a flexible manipulator. Mechatronics 15(10):1305-1320

10. Wai RJ, Lee M-C (2004) Intelligent optimal control of single-link flexible robot arm. IEEE Trans Ind Electron 51(1):201-220

11. Talebi HA, Khorasani K, Patel RV (1998) Neural network based control schemes for flexible-link manipulators: simulations and experiments, "neural net works", special issue. Neural Control Robotics Biol Technol 11:1357-1377

12. Moudgal VG, Passino KM, Yourkovitch S (1994) Rule-based control for flexible-link robot. IEEE Trans Control Syst Technol 2(4):393-405

13. Mannani A, Talebi HA (2007) A fuzzy-based Lyapunov-based control strategy for a macro-micro manipulator: experimental results. IEEE Trans Control Syst Technol 15(2):375-383

14. Lin J, Lewis FL (2002) Fuzzy-based control approach for flexible-link robot arm by reduced-order techniques. IEE Proc Control Theory Appl 149(3):177-187

15. Subudhi B, Morris AS (2003) Fuzzy-based and neuro-fuzzy-based approaches to control a flexible single-link manipulator. J Syst Control Eng Proc IMechE 217:387-399

16. Gutierrez LB, Lewis FL, Lowe JA (1998) Implementation of neural network tracking control approach for a single flexible link: comparison with PD and PID control approaches. IEEE Trans Ind Electron 45(2):307-318

17. Tian L, Wang J, Mao Z (2004) Constrained motion control of flexible robot manipulators based on recurrent neural networks. IEEE Trans Syst Man Cybern Part B Cybernetics 34(3):1541-1552

18. Caswara FM, Unbehauen H (2002) A neuro-fuzzy-based approach to the control of a flexible link manipulator. IEEE Trans Syst Man Cybern 18(6):932-944

19. Renno JM (2007) Inverse dynamics based tuning of a fuzzy-based control approach for a single link flexible manipulator. JVibr Control 13(12):1741-1759

20. Sarraf S, Fallah A, Seyedena T (2007) A neuro-fuzzy-based control approach for a single link flexible manipulator, Lecture notes in computer science, Artificial neural networks, ICANN 2007, vol. 4669. Springer, Berlin, pp 621-629

21. Lee CC (1990) Fuzzy-based in control systems—part I. IEEE Trans Syst Man Cybern 20(2):404-415

22. Moody J, Darken C (1989) Fast learning in networks of locally-tuned processing units. Neural Comput 1(2):281-294

23. Kennedy J, Eberhart RC (1995) Particle swarm optimization. In: Proceedings of IEEE international conference on neural networks, Perth, pp 1942-1948

24. Mote TP, Lokhande DS (2012) Temperature control system using ANFIS. Int J Soft Comput Eng 2(1):156-161

25. Mohammad MJ, Darus IZM (2014) Nonlinear system identification of vortex induced vibration on pipe cylinder. In: Proceedings of the 14th International Conference on Robotics, Control and Manufacturing Technology (ROCOM '14), Kuala Lumpur, Malaysia 23-25 April 2014

26. Bazargan A (2012) Design, development, and human analogous control of a climbing robot (Doctoral dissertation, Faculty of Graduate Studies and Research, University of Regina)

27. Manjaree S, Agarwal V, Nakra BC (2013) Inverse kinematic using neuro-fuzzy intelligent manipulator. Int J Adv Comput Res 3(4):160-165

28. Hsu F-C, Lin MC, Yeh RG (2013) Applied soft computing. Elsevier, Amsterdam, pp 1620-1626

29. Perng J-W, Chen G-Y, Hsieh S-C (2014) Optimal PID controller design based on PSO-RBF for wind turbine systems. Energies 7:191 